\title{
Schizophrenia: do all roads lead to dopamine or is this where they start? Evidence from two epidemiologically informed developmental rodent models
}

\author{
D Eyles $^{1,2}, \mathrm{~J} \mathrm{Feldon}^{3}$ and U Meyer ${ }^{3,4}$
}

The idea that there is some sort of abnormality in dopamine (DA) signalling is one of the more enduring hypotheses in schizophrenia research. Opinion leaders have published recent perspectives on the aetiology of this disorder with provocative titles such as 'Risk factors for schizophrenia-all roads lead to dopamine' or 'The dopamine hypothesis of schizophrenia-the final common pathway'. Perhaps, the other most enduring idea about schizophrenia is that it is a neurodevelopmental disorder. Those of us that model schizophrenia developmental risk-factor epidemiology in animals in an attempt to understand how this may translate to abnormal brain function have consistently shown that as adults these animals display behavioural, cognitive and pharmacological abnormalities consistent with aberrant DA signalling. The burning question remains how can in utero exposure to specific (environmental) insults induce persistent abnormalities in DA signalling in the adult? In this review, we summarize convergent evidence from two well-described developmental animal models, namely maternal immune activation and developmental vitamin $\mathrm{D}$ deficiency that begin to address this question. The adult offspring resulting from these two models consistently reveal locomotor abnormalities in response to DA-releasing or -blocking drugs. Additionally, as adults these animals have DA-related attentional and/or sensorimotor gating deficits. These findings are consistent with many other developmental animal models. However, the authors of this perspective have recently refocused their attention on very early aspects of DA ontogeny and describe reductions in genes that induce or specify dopaminergic phenotype in the embryonic brain and early changes in DA turnover suggesting that the origins of these behavioural abnormalities in adults may be traced to early alterations in DA ontogeny. Whether the convergent findings from these two models can be extended to other developmental animal models for this disease is at present unknown as such early brain alterations are rarely examined. Although it is premature to conclude that such mechanisms could be operating in other developmental animal models for schizophrenia, our convergent data have led us to propose that rather than all roads leading to DA, perhaps, this may be where they start.

Translational Psychiatry (2012) 2, e81; doi:10.1038/tp.2012.6; published online 21 February 2012

\section{Introduction}

That schizophrenia is considered a disorder of brain development is now no longer a matter of debate. Although symptom onset is in early adulthood, convergent evidence from the fields of epidemiology, neuroimaging and postmortem analysis strongly suggest that disruptions to early brain development are causal. ${ }^{1,2}$ However, the diverse array of adverse gestational events that have been associated with schizophrenia such as fetal hypoxia, obstetric complications, maternal infection/immune function, season of birth and maternal stress hampers the search for a common aetiology. ${ }^{3}$ The situation regarding the contribution of individual genes to schizophrenia is no less clear with at last count no fewer than
43 candidates having been proposed. ${ }^{4,5}$ Although polymorphisms within enzymes involved in dopamine (DA) turnover such as mono-amine oxidase $A$ and catechol-o-methyl transferase or DA 2 and 3 receptors rank highly among such candidates, none are significantly altered at the genome-wide level. ${ }^{5}$ However, the finding within the most recent Genome-Wide Association Study that patients may have alterations in a regulatory micro-RNA element for genes that control neuronal development may represent a real step forward in such studies. ${ }^{6}$

Although abnormalities in most neurotransmitter systems have been linked with this disorder, alterations in DA neurotransmission remain central to both its treatment and aetiological understanding. ${ }^{7}$ Hypotheses linking abnormal

\footnotetext{
${ }^{1}$ Queensland Brain Institute, University of Queensland, Brisbane, Queensland, Australia; ${ }^{2}$ Queensland Centre for Mental Health Research, The Park Centre for Mental Health, Wacol, Queensland, Australia; ${ }^{3}$ Laboratory of Behavioural Neurobiology, Swiss Federal Institute of Technology Zurich (ETH), Schwerzenbach, Switzerland and ${ }^{4}$ Physiology and Behaviour Laboratory, Swiss Federal Institute of Technology (ETH) Zurich, Schwerzenbach, Switzerland Correspondence: Dr D Eyles, Queensland Centre for Mental Health Research, Queensland Brain Institute, University of Queensland, St Lucia, Brisbane, QLD 4072 Australia.

E-mail: eyles@uq.edu.au; web address: www.qcmhr.uq.edu.au or www.qbi.uq.edu.au

Keywords: brain development; dopamine; maternal immune activation; ontogeny; schizophrenia; vitamin D

Received 26 October 2011; revised 13 December 2011; accepted 8 January 2012
} 
dopaminergic function and schizophrenia date from the seminal work of Carlsson and Lindquvist. ${ }^{8}$ Not surprisingly, in 50 years this hypothesis has been modified. The initial postulate that schizophrenia was a disorder of excess DA activity underwent a major revision in the early 1990s that attempted to incorporate positron emission tomography data that suggested there was hypofrontality in cortical regions. The proposal, bolstered by studies in animals, was that this hypofrontality was due to abnormally low dopaminergic signalling in cortical regions. ${ }^{9}$ This suggestion received direct support from the positron emission tomography studies of AbiDargham et al. ${ }^{10}$ who showed that DA (D1) receptors were increased in the dorso-lateral prefrontal cortex of patients with schizophrenia. This elevation in the primary DA receptor in the cortex correlated strongly with working memory deficits in these patients. Positron emission tomography studies, however, have provided a much more robust evidence base to support the original proposal that patients with schizophrenia have excessive subcortical dopaminergic activity with increased DA release, synthesis and storage. ${ }^{11-16}$

A common perspective that has emerged over the past few years is that DA abnormalities may represent an end state for multiple environmental and genetic factors that operate in unison to predispose an 'at risk' individual to symptomatic onset. This concept has been summarized in recent reviews with titles such as 'Schizophrenia: from developmental deviance to DA dysregulation', ${ }^{17}$ or 'Risk factors for schizophrenia-all roads lead to DA'. ${ }^{18}$ Perhaps even more succinctly, DA abnormalities in schizophrenia have been referred to as the so-called 'Final Common Pathway' or version III of the hypothesis. ${ }^{19}$ Although such perspectives are parsimonious in attempting to draw together the widely disparate data concerning the various risk factors and genes that contribute to this disorder, there is still the need to integrate dysfunctional DA signalling in the adult patient with the epidemiology indicating schizophrenia is a disorder of early brain development.

It is important here to consider the consequences of interfering with DA signalling during fetal brain development. Maternal use of agents that increase extrasynaptic DA such as cocaine or amphetamine can induce deficits in cognitive and attentional systems in children. ${ }^{20}$ Neuroimaging studies on such children also suggest these agents can alter DA receptor and transporter numbers and produce reductions in the volumes of major dopaminergic terminal fields like the striatum. $^{21,22}$ It would be of intense interest to follow-up psychiatric outcomes in such children.

An examination of the so-called 'prodromal stage' of the disease also provides evidence for underlying dopaminergic deficits in schizophrenia. In an important recent study, adults that were at risk but asymptomatic who later progressed to psychosis were shown to have increased subcortical DA uptake compared with controls, indicating dysfunction in DA signalling may be a precursor to schizophrenia. ${ }^{23}$

The obstacles to unravelling the shared mechanisms between the diverse genetic and environmental factors responsible for schizophrenia are considerable. First, there is no single exposure/gene variant or early diagnostic feature predictive for later onset of this disease. Second, the ability to study brain development in humans is obviously fraught with technical difficulties. Finally, even if the research community could agree on the worthiness of any early candidate exposure/s, such a research programme would need to be followed up for decades to correlate early life exposure with onset of symptoms, a course that is clearly not practical.

The use of neurodevelopmental animal models drastically reduces these ethical and temporal demands. ${ }^{24}$ Of the plethora of models have been developed (primarily in rodents) one feature that would appear to be germane would be an alteration of DA-related behaviours in the adult. Many of these models possess strong face and/or predictive validity for the disorder. ${ }^{25}$ In particular, prenatal exposure to the DNA methylation agent methylazoxymethanol acetate, and the postnatal ventral hippocampal lesion model produce adult animals that perhaps best phenocopy certain aspects of abnormal brain function in patients. ${ }^{26,27}$ Such phenotypes are attenuated by antipsychotics indicating these models also possess strong predictive validity. These models are now also providing some mechanistic understanding behind how developmental interventions can induce loss of cortical GABAergic interneuron function, a particularly prominent explanation for higher cortical dysfunction in schizophrenia. ${ }^{28}$ The work of O'Donnell ${ }^{29}$ and others is particularly important here as this group has shown that neonatal hippocampal lesions impair dopaminergic innervation of the prefrontal cortex during adolescence. This is therefore direct evidence that an early postnatal intervention that is believed relevant to schizophrenia can induce late (adolescence) developmental alterations in DA ontogeny. The perinatal hypoxia models designed to examine the effects of obstetric complications also produce abnormalities in offspring behaviour that implicate impaired DA signalling. ${ }^{30}$

Other models have even directly modified postnatal DA signalling via early transient alterations in D2 receptor density $^{31}$ or indirectly by exposing maternal animals to DAreleasing agents such as amphetamine. ${ }^{32}$ Not surprisingly, such exposures produce long-lasting changes in DA-related behaviours in the exposed offspring. An excellent register of all such models was prepared by Koenig et al. and is freely available http://www.schizophreniaforum.org/res/models/ default.asp.

Although such models are informative regarding the effect of early environment on brain development and function as yet they tell us little about how such adverse exposures could interact with genomic factors. Additionally, as useful as many of these models are for understanding the abnormal connectivity and brain function that may result from adverse developmental exposures, many of them, that is, neonatal ventral hippocampal lesion or prenatal methylazoxymethanol acetate exposure have no direct aetiological relationship with schizophrenia as patients have not been exposed to severe mitogens during development or have lesions within their hippocampus. The three authors of this current review have over the past 10 years been primarily responsible for the development of the developmental vitamin D (DVD)-deficiency and maternal immune activation (MIA) models, which are both featured in the aforementioned register. These models share both the face and predictive validity of many other developmental animal models but are based on epidemiologically proven risk factors. Independently, we have 
recently refocused our research efforts to concentrate on whether these exposures could produce any early neurobiological alterations. Our unique contribution to the field is the realization that these models show very early changes in the expression of genes that are either involved in the induction and/or specification of DA neurons or in the expression of enzymes responsible for DA turnover. We hypothesize that such early alterations subtly alter the ontogeny of DA neurons creating long-lasting changes in DA neuron connectivity, function and perhaps even survival. We consider it highly plausible that very early alterations in DA-mediated pathways may be induced in other developmental models for this disorder. Appropriately, the authors of animal models for schizophrenia have focused on replicating as much of the structural and behavioural phenotype in the adult offspring (for an excellent recent summary, see Brown ${ }^{33}$ ). However, most authors have completely ignored the developing brain in such studies at any time point proximal to the adverse maternal or postnatal exposure. Therefore, there is an absence of data concerning early aspects of DA or any other neurotransmitter ontogeny in such models. Our choice therefore to focus on data obtained from the DVD-deficiency and MIA models in this review is due to an absence of published evidence in other animal models. We think it entirely likely that early aspects of DA ontogeny may also be altered in other models. We believe that future studies directed toward understanding what common early mechanism/s underpin DA neuron dysfunction in the adult may represent a useful approach to understanding the aetiology of this disease.

\section{The DVD-deficiency and MIA developmental animal models of schizophrenia}

DVD-deficiency has been shown to be a direct risk factor for schizophrenia with an odds ratio of 2.0. ${ }^{34}$ There is also some indirect supporting evidence. ${ }^{35,36}$ The proposal that maternal vitamin $D$ deficiency could be a risk-modifying agent for schizophrenia was made 11 years ago. ${ }^{37}$ This led to the development of a rodent model restricting the period of vitamin $D$ deficiency to gestation. ${ }^{38}$ This model describes structural brain changes such as ventriculomegaly, ${ }^{38,39}$ baseline cognitive abnormalities in domains of attention ${ }^{40}$ and behavioural sensitivity to both $N$-methyl-D-aspartate antagonists and amphetamine, ${ }^{41-43}$ meaning this model possesses strong face and construct validity. Although the predictive validity of this model has been less well established, it has been explored in some detail by one group. In their first study, this group showed that haloperidol, a widely prescribed antipsychotic, could restore habituation deficits in an exploration paradigm. ${ }^{44}$ Next, this group demonstrated that DVDdeficiency altered hippocampal long-term potentiation, a neurobiological correlate of learning and memory. They showed that both risperidone and haloperidol normalized long-term potentiation in DVD-deficient offspring. ${ }^{45}$ Most recently, this group showed that haloperidol restored adult neurogenesis deficits in this model. ${ }^{46}$ Studies in other laboratories have also shown that haloperidol appeared to preferentially normalize $N$-methyl-D-aspartate antagonist induced hyperlocomotion in DVD-deficient rats compared with that induced in controls. ${ }^{41}$
MIA or prenatal exposure to infection are also wellestablished risk factors for schizophrenia. ${ }^{47,48}$ The relative odds risk of MIA induced by a variety of prenatal infections has been summarized in a recent review as being between 2-6 and 3.0 depending on the nature and timing of the infection. ${ }^{48}$

Animal models have been developed to explore the underlying neurobiology of this risk. ${ }^{49}$ One such MIA model makes use of prenatal maternal treatment with the viral mimic polyriboinosinic-polyribocytidilic acid (poly(l:C)), a synthetic analogue of double-stranded RNA that readily stimulates cytokine-associated viral-like acute phase responses in maternal and fetal compartments. A plethora of experimental studies have revealed that prenatal poly(I:C) treatment in mid-gestation (in mice typically $\sim$ gestation day 9 to 12 ; in rats typically $\sim$ gestation day 15) leads to the emergence of multiple behavioural, cognitive and pharmacological dysfunctions in adulthood, many of which are directly implicated in schizophrenia (reviewed in Meyer and Feldon $^{24}$ ). The spectrum of such dysfunctions includes abnormalities in sensorimotor gating, selective attention, social interaction, working memory and sensitivity to psychostimulant drugs. Many of the prenatal poly $(\mathrm{I}: \mathrm{C})$-induced functional abnormalities can be normalized by acute and/or chronic antipsychotic drug treatment and are dependent on post-pubertal maturational processes. ${ }^{50-55}$ The functional brain alterations in this MIA model are accompanied by a wide spectrum of neuroanatomical and neurotransmitter changes in the adult central nervous system, some of which are again highly relevant for schizophrenia, including presence of enlarged lateral ventricles, ${ }^{56,57}$ reduced hippocampal and prefrontal cortical expression of Parvalbumin and Reelin, ${ }^{58}$ and numerous DA abnormalities representative of an overactive striatal DA system. ${ }^{51-54}$ Taken together, the poly(I:C)-based MIA model in rats and mice is characterized by a high level of face, construct and predictive validity for schizophrenia-like pathology.

DVD-deficiency and MIA have also been implicated as risk factors for other psychiatric disorders such as autism. The evidence for DVD-deficiency, however, is at best indirect ${ }^{59}$ and often absent. ${ }^{60,61}$ Large cross-sectional association studies are still yet to be published. The evidence for MIA is slightly stronger. Children who develop autism have abnormalities in peripheral immune function ${ }^{62}$ and poly(l:C)-exposed mice develop abnormalities in cerebellar Purkinje cells similar to that seen in children with autism. ${ }^{63}$

\section{DVD-deficiency and DA dysfunction in adult offspring}

Early studies reported that rat weanlings deprived of dietary vitamin D had increased catecholamine levels in cortical and hypothalamic areas when examined as adults. ${ }^{64} \mathrm{~A}$ later study in vitamin D-deficient animals also provided some support for increased DA levels in striatum. ${ }^{65}$ However, unlike DVDdeficient rats, co-existing hypocalcaemia in both these early studies may confound any direct effect of vitamin D deficiency. Curiously, DA can also induce signalling at the vitamin $D$ receptor in the absence of the vitamin, suggesting a complex interaction between vitamin $D$ and catecholamines. ${ }^{66}$ Indirect indications that vitamin D may affect dopaminergic signalling also come from the recognition that 
the vitamin $D$ receptor and the enzyme responsible for the synthesis of the active form of the hormone CYP27B1 have a strong distribution in the DA-rich region of the human brain, the substantia nigra. ${ }^{67}$

Spontaneous hyperlocomotion in response to a novel environment is a common behavioural readout of an underlying hyperdopaminergia. When DVD-deficient rats are placed in a novel arena these animals display spontaneous hyperlocomotion without any anxiety or depressive-like phenotype. ${ }^{68}$ This is unlikely to be a stress-mediated mechanism because the animals have normal hypothalamic pituitary adrenal axis-mediated stress responses. ${ }^{69}$ These animals are also selectively sensitive to the locomotorenhancing effects of amphetamine, a drug that enhances DA release. ${ }^{43}$ A relationship between vitamin D and DA in vivo is further supported via experiments where the active form of vitamin $D, 1,25(\mathrm{OH})_{2} D_{3}$ is given to adult animals. When $1,25(\mathrm{OH})_{2} \mathrm{D}_{3}$ is administered long-term, increased basal striatal DA levels and increases in evoked release of DA result. ${ }^{70} 1,25(\mathrm{OH})_{2} \mathrm{D}_{3}$ has also been administered to newborn rats and DA and noradrenalin measured in a variety of brain regions in these animals as adults. These studies revealed that DA and noradrenalin were elevated mainly in the brainstem of these animals as adults. ${ }^{71}$

\section{MIA and DA dysfunction in adult offspring}

Prenatal immunological insults using agents such as poly(I:C) are capable of inducing long-lasting molecular and functional brain abnormalities in the adult, which are strongly associated with imbalances in the mesolimbic and/or mesocortical DA system. ${ }^{72}$ One of the well-established functional phenotypes displayed by adult poly $(\mathrm{I}: \mathrm{C})$ offspring is the potentiation of amphetamine sensitivity. ${ }^{51,53,54}$ Furthermore, adult offspring born to poly(I:C)-treated mothers show a DA-dependent disruption of selective attention and sensorimotor gating. Accordingly, DA receptor-blocking compounds can effectively normalize such deficits in adult poly(l:C) offspring. ${ }^{53-55}$ There is also robust evidence for the presence of long-term DArelated molecular alterations in the adult central nervous system of prenatally poly(l:C)-treated animals. First, similar to post-mortem findings in schizophrenic patients, ${ }^{73}$ these animals consistently show enhanced striatal expression of tyrosine hydroxylase $(\mathrm{TH})$, the rate-limiting enzyme in the biosynthesis of DA. ${ }^{51,54}$ Importantly, the implementation of neonatal cross-fostering procedures in the mouse poly $(\mathrm{I}: \mathrm{C})$ MIA model suggests that the effects of prenatal immune activation on altered striatal $\mathrm{TH}$ expression are attributable to prenatal but not postnatal maternal effects on the offspring. This is evidenced by enhanced striatal $\mathrm{TH}$ immunoreactivity being found in prenatally poly $(\mathrm{I}: \mathrm{C})$-treated offspring regardless of whether they were raised by surrogate dams, which had experienced poly $(\mathrm{I}: \mathrm{C})$ treatment during pregnancy, or whether they were adopted by sham-treated control surrogate dams. ${ }^{51}$ In addition to presynaptic dopaminergic changes, adult poly (I:C) offspring have also been reported to display DA receptor changes relevant to schizophrenia, including reduced densities of D1 receptors in prefrontal cortex and elevated densities of D2 receptors in the striatum. ${ }^{51,54}$ Finally, increased basal levels of the DA metabolite homovanillic acid and enhanced $\mathrm{KCl}$-induced $\mathrm{DA}$ release have been reported in striatal regions of adult poly(I:C)-exposed animals. ${ }^{53,74}$

Many of these DA-related molecular and behavioural abnormalities have also been reported in adult offspring from other developmental animal models for schizophrenia. ${ }^{75}$ However, new data from both the DVD-deficiency and MIA models are now indicating that there may be very early alterations in dopaminergic components in both these models.

\section{DVD-deficiency and DA ontogeny}

DVD-deficiency alters factors important in the ontogeny of embryonic rat dopaminergic neurons. The vitamin $D$ receptor initially appears in the developing brain on embryonic day 12 (E12), where it is most highly expressed in the neuroepithelium of the midbrain. ${ }^{76}$ Almost all mesencephalic DA (msDA) neurons are born between E11 and E14 in the SpragueDawley rat, with $80 \%$ of these cells being born between E11 and $\mathrm{E} 12 .{ }^{77} \mathrm{E} 12$ therefore potentially represents a critical time for the convergence between vitamin $D$ function in embryonic brain and DA ontogeny. To examine this link, a recent study examined DA-selective gene expression in embryonic mesencephalon at two specific ages, E12 representing the peak for msDA neuron proliferation, and E15, a developmental time point representing a largely post-proliferation period for msDA neurons. ${ }^{77}$ The most widely studied early specification factors for msDA neurons, (Nurr1, p57Kip2, Lmx1b and ptx3) were investigated. ${ }^{78} \mathrm{TH}$ expression was also quantified at these time points as a marker of dopaminergic phenotype. This study revealed that the crucial specification factor for dopaminergic phenotype Nurr 1 (also known as NR4A2 an orphan nuclear receptor) was reduced at $E 12$ in the mesencephalon of DVD-deficient rat embryos. A co-related factor, p57Kip2, was also reduced at this age. At the later embryonic age, E15, Nurr 1 expression was still reduced but p57Kip2 levels had normalized. Early markers for dopaminergic maturation such as $\mathrm{TH}$, also appeared to be reduced especially at the earlier age. ${ }^{79}$

DA levels have also been examined directly in the neonatal forebrains of DVD-deficient neonates. Although DA levels were shown to be normal, its metabolism was altered with increased ratios of 3,4-dihydroxyphenylacetic acid/homovanilic acid (two major DA metabolites). ${ }^{80}$ This was accompanied by a reduction in catechol-o-methyl transferase, the enzyme that converts 3,4-dihydroxyphenylacetic acid to homovanilic acid. Therefore, it would appear that in a normo-calcaemic environment vitamin $D$ deficiency may not affect DA levels in developing brains per se but may affect its turnover.

\section{MIA and DA ontogeny}

Changes in specification and induction factors specific for DA neurons have also been reported in the whole brains of embryos derived from gestational day 9 poly(l:C)-exposed mice. It is important to emphasize that a single maternal poly $(\mathrm{l}: \mathrm{C})$ administration regime induces a marked but highly time-limited inflammatory response in the maternal host, lasting only approximately $36-48 \mathrm{~h}^{49} \mathrm{~A}$ strength of the 
poly $(\mathrm{I}: \mathrm{C})$ model therefore is the narrow window of exposure meaning the adverse effects of inflammation can be studied at discrete gestational ages. In the first study that examined the effects of MIA on DA ontogeny, embryos were examined 2, 4 and 8 days after poly $(\mathrm{I}: \mathrm{C})$ exposure. The first period examined was $E 11$, which approximates the peak time for DA cell birth in the mouse. ${ }^{81}$ The later periods $\mathrm{E} 13$ and $\mathrm{E} 17$ reflect an immediate and a more distal post-mitotic stage for DA neurons in the mouse. ${ }^{82}$ This initial study described a complex dynamic relationship between early inductive factors such as Sonic hedge hog and fibroblast growth factor 8 (fgf 8), which were expressed at control or even elevated levels at E11 gradually reducing to below control levels by E17. ${ }^{83}$ This correlated inversely with an initial decrease in the expression of the important dopaminergic specification factors Nurr 1 and ptx 3 at $\mathrm{E} 11$ a finding that had normalized by the first postmitotic stage for DA neurons, E13.

It is interesting to note that at later embryonic stages such as E17 and E19, when msDA neurons are largely post-mitotic, embryos derived from poly(I:C)-exposed mice show a significant increase in the number of $\mathrm{TH}$ - and Nurr1-positive cells. ${ }^{54,83}$ Thus, it appears that subsequent to the initial decrease at early-to-mid embryonic stages, early gestational immune challenge induces an over compensation in the expression of specification and maturational dopaminergic markers at later stages of development. Therefore, the effects of MIA on dopaminergic systems are not static. Rather, these early-life insults induce primary changes in early embryonic DA development, which then interact with maturational processes to precipitate long-term dopaminergic abnormalities that are, at least in part, dependent on the actual stage of pre- and postnatal development.

\section{DVD-deficiency and MIA, early effects on DA ontogeny}

Taken together, there are some coherent patterns emerging within the DVD-deficiency and poly $(\mathrm{I}: \mathrm{C})$ models as regards to their effects at least on early phases of DA ontogeny. In both the DVD-deficient rat and the prenatal poly(I:C)-exposed mouse, there is an initial reduction in expression of factors crucial for specifying dopaminergic phenotype at times reflecting the peak period of DA cell birth in both species, $E 12$ and $E 11$, respectively. This abnormality in DA ontogeny is normalized somewhat when next examined at a period representing a largely post-mitotic phase for dopaminergic neurons. This correction is complete for Nurr 1 and ptx3 in the poly $(\mathrm{I}: \mathrm{C})$ model and partial for the DVD-deficiency model with p57Kip2 expression returning to control levels but Nurr 1 expression remaining reduced at E15. Later embryonic stages indicate that Nurr 1even appears to be increased in the poly(I:C)-exposed brains. Such stages in embryonic mesencephalic development have yet to be examined in the DVD-deficient embryos.

Is there evidence for a direct mechanism? Despite the collective data presented that suggests both models alter factors important in DA ontogeny, evidence for a direct mechanism is still lacking. Direct links have been made between vitamin $\mathrm{D}$ enhancing the production of $\mathrm{TH}$ both in vitro $^{84}$ and in certain in vivo pathological models. ${ }^{85-87}$
Vitamin D may also increase $\mathrm{TH}$ indirectly by promoting glial-derived neurotrophic factor production. ${ }^{88}$ Glial-derived neurotrophic factor activates $\mathrm{TH}$ by phosphorylating this enzyme in DA-rich brain regions. ${ }^{89}$ The developmental absence of vitamin $\mathrm{D}$ therefore is consistent with a reduction in $\mathrm{TH}$ in utero $^{79}$ but evidence for a direct mechanism is still lacking.

Poly(I:C) releases a cascade of pro-inflammatory cytokines, which are likely to cross the placenta and infiltrate the developing brain. ${ }^{90}$ Cytokines released after poly(I:C) exposure include the pro-inflammatory cytokine interleukin-1 $\beta$, which is known to exert multiple direct effects on DA development and/or maturation, including promotion of DA phenotypes in mesencephalic progenitor cells ${ }^{91-93}$ and enhancement of fetal DA cell survival. ${ }^{94}$ Mitotic progenitor cells isolated from the embryonic rat mesencephalon are also known to potently respond to a combination of interleukin-1, interleukin-11, leukaemia inhibitory factor and glial-derived neurotrophic factor promoting cells toward a dopaminergic phenotype. ${ }^{93}$ A cytokine-enhanced survival of dopaminergic cell types would appear to contrast with the initial reduction in markers for dopaminergic phenotype seen in poly(I:C)-treated embryos. However, with regards to direct cytokine effects on DA development, it is important to emphasize that the responsiveness and/or sensitivity of developing cells to (proinflammatory) cytokines can vary considerably with neurodevelopmental stage. ${ }^{95}$ For example, while the pro-inflammatory cytokine tumour necrosis factor- $\alpha$ is neurotrophic to dopaminergic ventral mesencephalic neurons during early fetal development, the same molecule can exert neurotoxic effects on these cells at later stages of fetal brain development. ${ }^{96,97}$ Therefore, while it remains entirely plausible that poly(I:C)induced alterations in inflammatory cytokines may be responsible for early alterations in DA ontogeny, the timing of such alterations must be strictly observed before a direct mechanism could be postulated.

Evidence for an indirect mechanism. The alteration in the temporal pattern of DA-related gene expression in both models is highly suggestive that they may occur secondary to a delay in DA neuron maturation. Such a proposal is consistent with both the knowledge that vitamin $D$ is a critical differentiation factor in numerous cell types; ${ }^{98}$ and the observation that cytokine-associated inflammatory events in the early fetal brain primarily affect cell proliferation and differentiation. ${ }^{99}$

If vitamin $D$ is removed during development, cell proliferation is enhanced across most brain regions ${ }^{38,100}$ with corresponding changes in cell-cycle and apoptotic gene expression. ${ }^{100}$ Progenitor cell formation is also enhanced in these brains. ${ }^{101}$ This finding can also be reversed via the addition of $1,25(\mathrm{OH})_{2} \mathrm{D}_{3} .{ }^{101}$ Additionally, adding $1,25(\mathrm{OH})_{2} \mathrm{D}_{3}$ to primary hippocampal cells in culture differentiates them by decreasing the number of proliferating cells and increasing neurite outgrowth. ${ }^{102}$ On the balance of this evidence, therefore, it would appear that DVD-deficient embryonic brains are less differentiated. Given that embryonic DA cells immediately begin to express Nurr1 only once progenitor cells stop proliferating ${ }^{103}$ and $8 \mathrm{~h}$ later p57Kip2 (ref. 104) one would predict that the expression of these factors would be delayed or decreased in DVD-deficient mesencephalon. The high 
density of receptors for vitamin $D$ in the developing mesencephalon at E12 further suggests that this region could be particularly sensitive to vitamin D status. ${ }^{76}$

Similarly, in the poly $(\mathrm{l}: \mathrm{C})$ model an initial increase in the expression of induction factors such as fgf 8 at the peak period of DA neuron birth ( $E 11$ in the mouse) is suggestive of enhanced proliferation and a delay in differentiation. ${ }^{83}$ This finding is internally consistent with the initial decrease or delay in the expression of post-mitotic factors such as Nurr 1 and ptx 3 in these same mouse embryos. The normalization of Nurr 1 and ptx 3 expression in poly(I:C)-treated embryonic brains by E13 (a largely post-mitotic stage for DA neurons in the mouse) correlates with a rapid decrease in the expression of both fgf 8 and Sonic hedge hog (another important induction gene for DA neurons) indicating DA cells have ceased dividing. ${ }^{82}$ Unfortunately, these same early induction factors have not yet been studied in the DVD-deficiency model. Nevertheless, vitamin D3 is known to suppress the transcription of fgf $8 .{ }^{105}$ Therefore, it is plausible that the expression of fgf 8 may also be elevated in the DVD-deficient brain.

Although a delay in the development of dopaminergic systems have been reported here, we cannot rule out alterations in the early maturation of other neurotransmitter systems in both models. For instance, abnormalities in selective aspects of GABA signalling have been reported to be altered in adult offspring in both models. ${ }^{39,106}$ However, it must be noted that cortical GABAergic systems develop later than dopaminergic systems, therefore such changes may well occur secondary to a primary developmental DA disruption. Additionally, a study of neuroactive amines in DVD-deficient neonatal brains revealed that DA alone was altered when compared with noradrenalin and serotonin. ${ }^{80}$ Unfortunately, no similar studies have been conducted in poly(I:C)-treated brains.

\section{What are the consequences of altered DA ontogeny for the developing brain?}

Alterations in early induction factors such as fgf 8 , Sonic hedge hog; specification factors for dopaminergic phenotype such as Nurr 1, p57Kip2 and ptx3; and the reduction of enzymes crucial for DA turnover such as catechol-o-methyl transferase in the embryonic brain are likely to be highly relevant for developing msDA neurons. Fgf 8 is secreted from the rostral hindbrain early in development and is essential for the orderly formation of the boundary between mid and hindbrain. ${ }^{107}$ In particular, it would appear that fgf 8 is essential for the proper positioning of mitotic cells within this region many of which will become dopaminergic. ${ }^{78}$ Nurr1 is an obligatory factor in msDA neuron development and maturation. ${ }^{108}$ Studies have convincingly shown that Nurr1-/- transgenic animals have complete agenesis of DA neurons, whereas other monoamine neurons such as serotonin and noradrenaline are unaffected. ${ }^{109}$ Nurr1 also has a number of functions in post-mitotic and mature msDA neurons. ${ }^{82,110}$ It regulates DA synthesis via $\mathrm{TH}$, vesicular content via the transporter VMAT2, release via the DA transporter and trophic support via regulation of the receptor for glial-derived neurotrophic factor, c-RET. ${ }^{78}$ The long-term consequences of Nurr 1 ablation cannot be studied in the homozygote as this deletion is lethal, but in the heterozygote this leads to a reduction in TH-positive neurons. ${ }^{111}$

Nurr1 and p57kip2 expression appear to be closely linked. ${ }^{82}$ p57kip2 is drastically reduced in Nurr1 $+/$ - embryonic brains. p57Kip2 co-immunoprecipitates with Nurr1 when Nurr1 antibodies are used in disassociated rat mesencephalon. ${ }^{103}$ Therefore, it would appear that Nurr1 and p57kip2 co-operate to regulate DA cell differentiation and maturation. p57Kip2, however, is also an important agent independent of Nurr 1 in DA ontogeny. p57Kip2 null mutant mice have no TH-positive mesencephalic cells at E18.5. ${ }^{104}$ Although p57kip2 is a cyclindependent kinase inhibitor and therefore likely to have some effect on cell-cycle it would appear that at least in the embryonic mesencephalon it is primarily involved in post-mitotic functions such as cell migration and survival. ${ }^{103,104}$ The genetic ablation of either Nurr1 or p57Kip2 both lead to a preferential reduction in lateral migration of msDA neurons, ${ }^{103,108,110}$ that is, those neurons most likely to form the embryonic substantia nigra. Whether this affects cell number within the ventral tegmental area is unknown. Additionally, the timing of cell division also influences eventual cell positioning with neurons that cease division early migrating most rostrally in the mesencephalon. ${ }^{112}$ Thus, the combination of delayed differentiation and reduced expression of factors such as p57Kip2 in DVD-deficient mesencephalon and Nurr 1 at least initially in both models could likely lead to abnormal rostral/lateral migration of msDA neurons with the downstream effects on dopaminergic connectivity.

ptx 3 is another specification agent for DA neurons being specifically involved in terminally differentiating DA neurons. ${ }^{113} \mathrm{ptx} 3$ acts co-operatively with Nurr 1 in post-mitotic $\mathrm{DA}$ neurons to achieve this. ${ }^{114}$ In the poly(l:C)-exposed embryo, expression of ptx 3 mimicked that of Nurr 1. Finally, alterations in enzymes such as catechol-o-methyl transferase with concomitant alterations in DA turnover could also reflect early changes in DA innervation and/or signalling. The molecular alterations induced by DVD-deficiency or MIA in factors involved in these early aspects of msDA neuron ontogeny are summarized in Figure 1.

Taken together, the evidence obtained from both these developmental models indicate that there are early alterations in the expression of genes involved in the induction, positioning and differentiation of dopaminergic neurons, however, many questions remain. We know very little about how such early alterations could affect DA cell maturation processes in developing mesencephalon or how these changes could lead to abnormal dopaminergic signalling in the adult. Indeed, apart from a modest volume of literature dealing with the long-term effects of reduced Nurr 1 (see below), there are few studies that have examined adult brain function in models of compromised dopaminergic ontogeny. ${ }^{54}$ Moreover, apart from the transient D2 overexpressing mouse (see below), ${ }^{31}$ studies that examine adult brain function in models where interventions are restricted to early critical periods in DA ontogeny are conspicuous by their absence.

\section{What are the consequences of altered DA ontogeny for adult brain function?}

Studies concentrating on the selective modulation of early genes crucial in specifying dopaminergic phenotype and what 


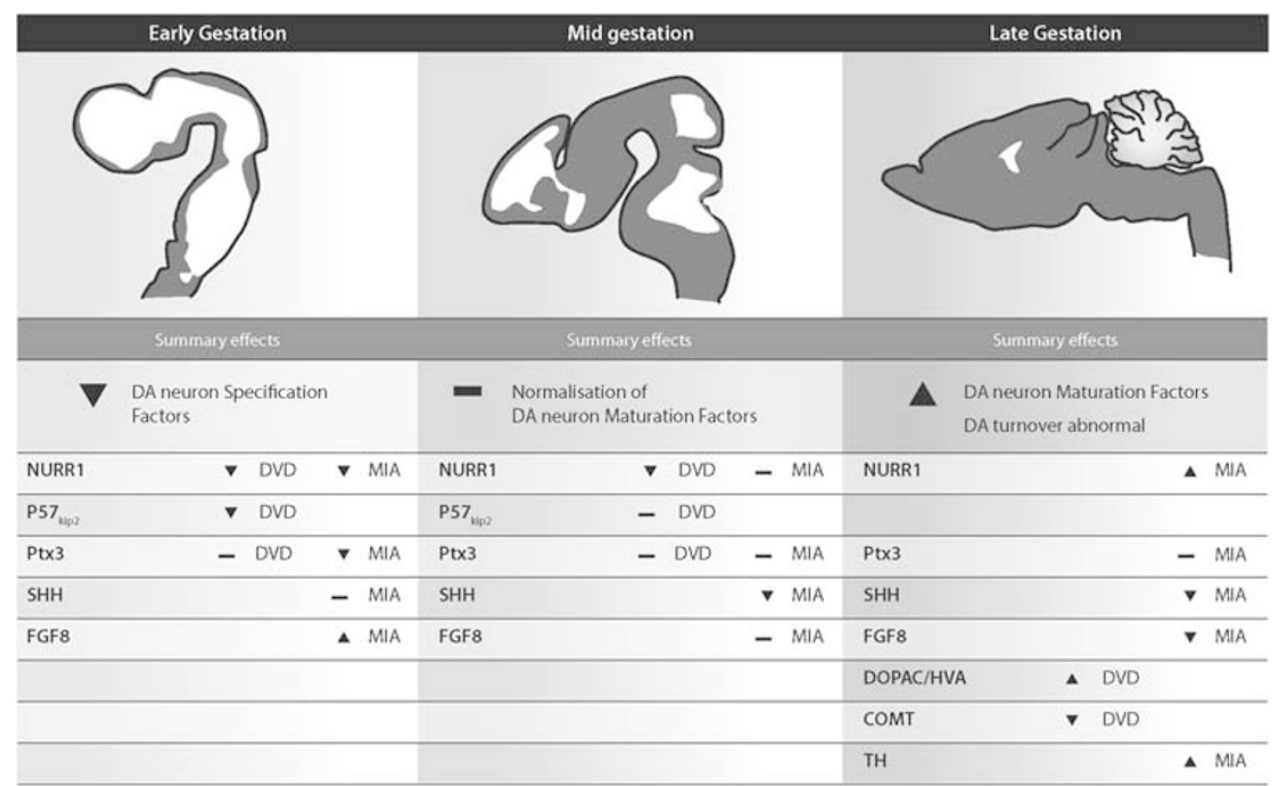

Figure 1 A summary of factors involved in the induction, specification and maturation of dopaminergic (DA) neurons affected by either developmental vitamin D (DVD)deficiency or maternal immune activation (MIA) at various stages of embryonic brain development. ( $\boldsymbol{\Delta}$ Increase in expression; $\boldsymbol{\nabla}$ decrease in expression; $\boldsymbol{\nabla}$ expression normalized or unaltered). COMT, catechol-o-methyl transferase; DOPAC, 3,4-dihydroxyphenylacetic acid; FGF8, fibroblast growth factor 8; HVA, homovanilic acid; SHH, Sonic hedge hog; $\mathrm{TH}$, tyrosine hydroxylase.

the downstream consequences for adult brain function may be are in their infancy. One of the most well-studied factors to date is Nurr 1. Adult Nurr1 $+/-$ mice have (a) spontaneous locomotion in response to novelty; $;^{115-117}$ (b) enhanced locomotion in response to both amphetamine ${ }^{115,117}$ and $\mathrm{N}$-methyl-D-aspartate antagonists; ${ }^{117}$ and (c) increased sensitivity to the locomotor retarding properties of haloperidol. ${ }^{117}$ This behavioural phenotype has led to the proposal that the Nurr1 + / mouse may be a viable animal model of schizophrenia. ${ }^{117}$ As previously discussed, this behavioural phenotype seen in Nurr 1 heterozygotes has been reproduced in both DVD-deficient offspring ${ }^{41-43,68}$ and poly(l:C)-treated offspring. ${ }^{51-55} \mathrm{~A}$ direct link between changes in these maturation factors for DA neurons and schizophrenia has also been made. A reduction in Nurr1 in the brains of patients with schizophrenia has been described. ${ }^{118}$ Polymorphisms in ptx 3 and other specification agents such as LMX1B have also been reported in patients with schizophrenia. ${ }^{119}$ Current investigation of ptx 3 and brain function is hampered by the fact that ptx3 transgenic mice do not respond to either DA agonists or antagonists. ${ }^{120}$

Although the use of transgenics in the study of schizophrenia is common, there is far less work using mutant strains where gene expression is conditional. Elucidating the 'when and where' of gene expression, especially during neurodevelopment will undoubtedly advance our understanding of the complex mechanisms within schizophrenia. Unfortunately, researchers interested in modelling the psychopathology of diseases such as schizophrenia all too rarely investigate the effects of transiently altering the expression of factors important to the developing brain. The approach of Kellendonk et al. ${ }^{31}$ is probably best known here. This group generated mice that express increased levels of $D 2$ receptors in a spatially restricted and temporally controlled manner. This group were able to verify that prolonged alteration in DA signalling throughout development and post-natal stages led to altered behaviours even when receptor density was normalized in the adult. Similar inducible strategies have been tried with disrupted in schizophrenia 1, reviewed in Pletnikov. ${ }^{121}$

Generation of these models, however, is time consuming, costly and currently restricted to single genes. The more recent advent of RNA interference technology has allowed investigators to achieve local reductions in gene expression in discrete brain nuclei. This technology is only now being applied to altering the expression of genes of intense interest to schizophrenia researchers such as disrupted in schizophrenia 1 (ref. 122) and D-amino acid oxidase. ${ }^{123}$ Despite these successes, the spatial and temporal control of gene expression in the developing or adult rodent brain remains technologically challenging.

These issues are drastically simplified in less complex organisms. For instance, the manipulation of gene-expression in the larval Zebra Fish (Danio Reio) brain via Morpholino RNA interference techniques is routine. Moreover, some groups have already described persistent functional consequences for DA-mediated behaviours in Zebra Fish in which Nurr 1 expression was reduced early in development. ${ }^{124}$ Drosophila melanogaster is even more accessible as a species for such investigation. For instance, the insertion of disrupted in schizophrenia 1 into Drosophila has recently been studied as an animal model of schizophrenia. ${ }^{125}$ Additionally, the variety of techniques to induce or silence gene expression and the strict temporal control of such gene-switching would appear to only be limited by the investigators imagination. The schizophrenia research community also would appear to be now considering this approach. ${ }^{126}$ 


\section{Inflammation and brain development: can DVD- deficiency and MIA be linked as risk factors for schizophrenia?}

There is clinical evidence to support the proposal that DVDdeficiency and MIA represent independent risk-modifying factors in schizophrenia. However, what may be less appreciated is that there could be some interaction between these non-genetic risk factors. There is now widespread agreement that vitamin $\mathrm{D}$ is a potent immunosuppressant. ${ }^{127}$ A number of studies have shown vitamin $D$ to be a potent suppressor of the types of inflammatory cytokines produced by MIA. This has led to the exploration of vitamin $D$ in certain models of brain inflammation. ${ }^{128}$ Although the combined effects of vitamin $D$ deficiency and poly $(\mathrm{l}: \mathrm{C})$ have not yet been investigated in the brain, the effects of another widely used inflammatory agent, lipopolysaccharide has. Lipopolysaccharide preferentially targets the Toll-4 receptor to elicit its inflammatory cascade and vitamin $D$ is known to directly antagonize this receptor. ${ }^{129}$ Accordingly, the co-administration of $1,25(\mathrm{OH})_{2} \mathrm{D}_{3}$ with this inflammatory agent in rat hippocampus leads to a recruitment of macrophages, a reduction in nitric oxide synthesis and preservation of hippocampal neuronal number. ${ }^{130}$ Studies in the developing brain examining whether the presence or absence of this vitamin will accordingly suppress or enhance outcomes mediated by inflammatory cytokines released by either poly $(\mathrm{I}: \mathrm{C})$ or lipopolysaccharide are now required.

\section{Conclusions}

Conceptually, the research community has moved on from considering schizophrenia the result of some static brain lesion whereby a single pathological process is responsible for the diverse symptomology observed. We now know that the mature brain is neither static in regards to cell number (neurogenesis) nor synaptic remodelling (experiencemediated plasticity). These factors are likely to be even more prescient in development. Longitudinal studies in adolescents at high risk of developing schizophrenia tend to indicate that any anatomical brain alterations occur before symptoms, are progressive and perhaps the result of enhanced endogenous processes rather than due to any external effect. ${ }^{131}$ The ethical and temporal demands make studying the aetiology of schizophrenia in patients an incredibly difficult undertaking. Therefore, as researchers we turn to animal models in an attempt to address neurobiological causality.

The two models we have focussed on here are based on plausible risk factor epidemiology for schizophrenia and have provided convergent evidence for a common mechanism that can integrate the two major hypotheses of schizophrenia to date, namely, that it is a neurodevelopmental disorder and that abnormalities in DA signalling are central. We speculate that early alterations in the trajectory of DA neuron development may also operate in other developmental models for this disorder. ${ }^{132}$ Given the common phenotype of DA dysfunction in most of these models it is somewhat perplexing that authors rarely, if ever, examine DA-related factors in the developing brain. On the basis of the data presented here, we propose that schizophrenia may be a disorder of abnormal DA ontogeny whereby multiple environmental factors subtly alter the trajectory of DA neuron birth, positioning and connectivity, in short, the ontogeny of DA neurons. How these early alterations can create the structural brain changes that are believed to be responsible for abnormal DA-mediated behaviours is now a key focus for our research. It is our hope that these recent findings in both the DVD-deficiency and MIA models may prompt other groups to re-investigate dopaminergic systems earlier during development than previously considered in an attempt to verify whether early alterations in DA ontogeny represent a shared aetiological pathway in animal models of schizophrenia.

\section{Conflict of interest}

The authors declare no conflict of interest.

Acknowledgements. DE acknowledges funding from the National Health and Medical Research Council of Australia and the Queensland State Government. UM and JF acknowledge funding from the Swiss National Science Foundation.

1. McGrath JJ, Feron FP, Burne TH, Mackay-Sim A, Eyles DW. The neurodevelopmental hypothesis of schizophrenia: a review of recent developments. Ann Med 2003; 35: 86-93.

2. Weinberger DR. Implications of normal brain development for the pathogenesis of schizophrenia. Arch Gen Psychiatry 1987; 44: 660-669.

3. van Os J, Kenis G, Rutten BP. The environment and schizophrenia. Nature 2010; 468: 203-212.

4. McClellan J, King MC. Genetic heterogeneity in human disease. Cell 2010; 141: 210-217.

5. Need AC, Ge D, Weale ME, Maia J, Feng S, Heinzen EL et al. A genome-wide investigation of SNPs and CNVs in schizophrenia. PLoS Genet 2009; 5: e1000373.

6. Ripke S, Sanders AR, Kendler KS, Levinson DF, Sklar P, Holmans PA et al. Genomewide association study identifies five new schizophrenia loci. Nat Genet 2011; 43: 969976.

7. Toda M, Abi-Dargham A. Dopamine hypothesis of schizophrenia: making sense of it all. Curr Psychiatry Rep 2007; 9: 329-336.

8. Carlsson A, Lindqvist $M$. Effect of chlorpromazine or haloperidol on formation of 3 methoxytyramine and normetanephrine in mouse brain. Acta Pharmacol Toxicol (Copenh) 1963; 20: 140-144.

9. Davis KL, Kahn RS, Ko G, Davidson M. Dopamine in schizophrenia: a review and reconceptualization. Am J Psychiatry 1991; 148: 1474-1486.

10. Abi-Dargham A, Mawlawi O, Lombardo I, Gil R, Martinez D, Huang $Y$ et al. Prefrontal dopamine D1 receptors and working memory in schizophrenia. J Neurosci 2002; 22: 3708-3719.

11. Kapur S, Mamo D. Half a century of antipsychotics and still a central role for dopamine D2 receptors. Prog Neuropsychopharmacol Biol Psychiatry 2003; 27: 1081-1090.

12. Breier A, Su TP, Saunders R, Carson RE, Kolachana BS, de Bartolomeis A et al. Schizophrenia is associated with elevated amphetamine-induced synaptic dopamine concentrations: evidence from a novel positron emission tomography method. Proc Natl Acad Sci USA 1997; 94: 2569-2574.

13. Abi-Dargham A, Gil R, Krystal J, Baldwin RM, Seibyl JP, Bowers M et al. Increased striatal dopamine transmission in schizophrenia: confirmation in a second cohort. $A m \mathrm{~J}$ Psychiatry 1998; 155: 761-767.

14. Lindstrom LH, Gefvert O, Hagberg G, Lundberg T, Bergstrom M, Hartvig P et al. Increased dopamine synthesis rate in medial prefrontal cortex and striatum in schizophrenia indicated by L-(beta-11C) DOPA and PET. Biol Psychiatry 1999; 46: 681-688.

15. Laruelle M, Abi-Dargham A, van Dyck CH, Gil R, D'Souza CD, Erdos J et al. Single photon emission computerized tomography imaging of amphetamine-induced dopamine release in drug-free schizophrenic subjects. Proc Natl Acad Sci USA 1996; 93: 92359240.

16. Lyon GJ, Abi-Dargham A, Moore H, Lieberman JA, Javitch JA, Sulzer D. Presynaptic regulation of dopamine transmission in schizophrenia. Schizophr Bull 2011; 37: 108-117.

17. Murray RM, Lappin J, Di Forti M. Schizophrenia: from developmental deviance to dopamine dysregulation. Eur Neuropsychopharmacol 2008; 18(Suppl 3): S129-S134.

18. Di Forti M, Lappin JM, Murray RM. Risk factors for schizophrenia-all roads lead to dopamine. Eur Neuropsychopharmacol 2007; 17(Suppl 2): S101-S107.

19. Howes OD, Kapur S. The dopamine hypothesis of schizophrenia: version III-the final common pathway. Schizophr Bull 2009; 35: 549-562. 
20. Thompson BL, Levitt $P$, Stanwood GD. Prenatal exposure to drugs: effects on brain development and implications for policy and education. Nat Rev Neurosci 2009; 10: 303312.

21. Chang L, Alicata D, Ernst T, Volkow N. Structural and metabolic brain changes in the striatum associated with methamphetamine abuse. Addiction 2007; 102(Suppl 1): 16-32.

22. Chang L, Smith LM, LoPresti C, Yonekura ML, Kuo J, Walot I et al. Smaller subcortical volumes and cognitive deficits in children with prenatal methamphetamine exposure. Psychiatry Res 2004; 132: 95-106.

23. Howes OD, Montgomery AJ, Asselin MC, Murray RM, Valli I, Tabraham P et al. Elevated striatal dopamine function linked to prodromal signs of schizophrenia. Arch Gen Psychiatry 2009; 66: 13-20.

24. Meyer U, Feldon J. Epidemiology-driven neurodevelopmental animal models of schizophrenia. Prog Neurobiol 2010; 90: 285-326.

25. Sams-Dodd F, Lipska BK, Weinberger DR. Neonatal lesions of the rat ventral hippocampus result in hyperlocomotion and deficits in social behaviour in adulthood. Psychopharmacology (Berl) 1997; 132: 303-310.

26. Tseng KY, Chambers RA, Lipska BK. The neonatal ventral hippocampal lesion as a heuristic neurodevelopmental model of schizophrenia. Behav Brain Res 2009; 204: 295 305

27. Lodge DJ, Behrens MM, Grace AA. A loss of parvalbumin-containing interneurons is associated with diminished oscillatory activity in an animal model of schizophrenia. J Neurosci 2009; 29: 2344-2354.

28. Hoftman GD, Lewis DA. Postnatal developmental trajectories of neural circuits in the primate prefrontal cortex: identifying sensitive periods for vulnerability to schizophrenia. Schizophr Bull 2011; 37: 493-503.

29. O'Donnell P. Adolescent onset of cortical disinhibition in schizophrenia: insights from animal models. Schizophr Bull 2011; 37: 484-492

30. Boksa P. Animal models of obstetric complications in relation to schizophrenia. Brain Res Brain Res Rev 2004; 45: 1-17.

31. Kellendonk C, Simpson EH, Polan HJ, Malleret G, Vronskaya S, Winiger V et al. Transient and selective overexpression of dopamine D2 receptors in the striatum causes persistent abnormalities in prefrontal cortex functioning. Neuron 2006; 49: 603-615.

32. Slamberova R, Pometlova M, Charousova P. Postnatal development of rat pups is altered by prenatal methamphetamine exposure. Prog Neuropsychopharmacol Biol Psychiatry 2006; 30: 82-88.

33. Brown AS. The environment and susceptibility to schizophrenia. Prog Neurobiol 2011; 93 23-58.

34. McGrath JJ, Eyles DW, Pedersen CB, Anderson C, Ko P, Burne TH et al. Neonatal vitamin $D$ status and risk of schizophrenia: a population-based case-control study. Arch Gen Psychiatry 2010; 67: 889-894.

35. McGrath J, Saari K, Hakko H, Jokelainen J, Jones $P$, Jarvelin MR et al. Vitamin D supplementation during the first year of life and risk of schizophrenia: a Finnish birth cohort study. Schizophr Res 2004; 67: 237-245.

36. McGrath JJ, Burne TH, Feron F, Mackay-Sim A, Eyles DW. Developmental vitamin D deficiency and risk of schizophrenia: a 10-year update. Schizophr Bull 2010; 36: 10731078

37. McGrath J. Hypothesis: is low prenatal vitamin D a risk-modifying factor for schizophrenia? Schizophr Res 1999; 40: 173-177.

38. Eyles D, Brown J, Mackay-Sim A, McGrath J, Feron F. Vitamin D3 and brain development. Neuroscience 2003; 118: 641-653.

39. Feron F, Burne TH, Brown J, Smith E, McGrath JJ, Mackay-Sim A et al. Developmental vitamin D3 deficiency alters the adult rat brain. Brain Res Bull 2005; 65: 141-148.

40. Becker A, Eyles DW, McGrath JJ, Grecksch G. Transient prenatal vitamin D deficiency is associated with subtle alterations in learning and memory functions in adult rats. Behav Brain Res 2005; 161: 306-312.

41. Kesby JP, Burne TH, McGrath JJ, Eyles DW. Developmental vitamin D deficiency alters MK 801-induced hyperlocomotion in the adult rat: An animal model of schizophrenia. Bio Psychiatry 2006; 60: 591-596.

42. O'Loan J, Eyles DW, Kesby J, Ko P, McGrath JJ, Burne TH. Vitamin D deficiency during various stages of pregnancy in the rat; its impact on development and behaviour in adult offspring. Psychoneuroendocrinology 2007; 32: 227-234.

43. Kesby JP, Cui X, O'Loan J, McGrath JJ, Burne TH, Eyles DW. Developmental vitamin D deficiency alters dopamine-mediated behaviors and dopamine transporter function in adult female rats. Psychopharmacology (Berl) 2010; 208: 159-168.

44. Becker A, Grecksch G. Pharmacological treatment to augment hole board habituation in prenatal Vitamin D-deficient rats. Behav Brain Res 2006; 166: 177-183.

45. Grecksch G, Ruthrich $H$, Hollt V, Becker A. Transient prenatal vitamin D deficiency is associated with changes of synaptic plasticity in the dentate gyrus in adult rats. Psychoneuroendocrinology 2009; 34(Suppl 1): S258-S264.

46. Keilhoff $G$, Grecksch $G$, Becker A. Haloperidol normalized prenatal vitamin D depletioninduced reduction of hippocampal cell proliferation in adult rats. Neurosci Lett 2010; 476 94-98.

47. Brown AS. Prenatal infection as a risk factor for schizophrenia. Schizophr Bull 2006; 32 200-202.

48. Brown AS, Derkits EJ. Prenatal infection and schizophrenia: a review of epidemiologic and translational studies. Am J Psychiatry 2010; 167: 261-280.
49. Meyer U, Feldon J, Fatemi SH. In-vivo rodent models for the experimental investigation of prenatal immune activation effects in neurodevelopmental brain disorders. Neurosci Biobehav Rev 2009; 33: 1061-1079.

50. Meyer U, Knuesel I, Nyffeler M, Feldon J. Chronic clozapine treatment improves prenatal infection-induced working memory deficits without influencing adult hippocampal neurogenesis. Psychopharmacology (Berl) 2010; 208: 531-543.

51. Meyer U, Nyffeler M, Schwendener S, Knuesel I, Yee BK, Feldon J. Relative prenatal and postnatal maternal contributions to schizophrenia-related neurochemical dysfunction after in utero immune challenge. Neuropsychopharmacology 2008; 33: 441-456.

52. Ozawa K, Hashimoto K, Kishimoto T, Shimizu E, Ishikura H, lyo M. Immune activation during pregnancy in mice leads to dopaminergic hyperfunction and cognitive impairment in the offspring: a neurodevelopmental animal model of schizophrenia. Biol Psychiatry 2006; 59: 546-554.

53. Zuckerman L, Weiner I. Post-pubertal emergence of disrupted latent inhibition following prenatal immune activation. Psychopharmacology (Berl) 2003; 169: 308-313.

54. Vuillermot S, Weber L, Feldon J, Meyer U. A longitudinal examination of the neurodevelopmental impact of prenatal immune activation in mice reveals primary defects in dopaminergic development relevant to schizophrenia. J Neurosci 2010; 30: 1270-1287.

55. Zuckerman L, Weiner I. Maternal immune activation leads to behavioral and pharmacological changes in the adult offspring. J Psychiatr Res 2005; 39: 311-323.

56. Li Q, Cheung C, Wei R, Hui ES, Feldon J, Meyer $U$ et al. Prenatal immune challenge is an environmental risk factor for brain and behavior change relevant to schizophrenia: evidence from MRI in a mouse model. PLoS One 2009; 4: e6354.

57. Piontkewitz $Y$, Assaf $Y$, Weiner I. Clozapine administration in adolescence prevents postpubertal emergence of brain structural pathology in an animal model of schizophrenia. Biol Psychiatry 2009; 66: 1038-1046.

58. Meyer U, Nyffeler M, Yee BK, Knuesel I, Feldon J. Adult brain and behavioral pathological markers of prenatal immune challenge during early/middle and late fetal development in mice. Brain Behav Immun 2008; 22: 469-486.

59. Humble MB, Gustafsson S, Bejerot S. Low serum levels of 25-hydroxyvitamin D (25$\mathrm{OHD)}$ among psychiatric out-patients in Sweden: Relations with season, age, ethnic origin and psychiatric diagnosis. J Steroid Biochem Mol Biol 2010; 121: 467-470.

60. Fernell E, Barnevik-Olsson M, Bagenholm G, Gillberg C, Gustafsson S, Saaf M. Serum levels of 25-hydroxyvitamin $D$ in mothers of Swedish and of Somali origin who have children with and without autism. Acta Paediatr 2010; 99: 743-747.

61. Eyles DW. Vitamin D and autism: does skin colour modify risk? Acta Paediatr 2010; 99 : 645-647.

62. Enstrom AM, Onore CE, Van de Water JA, Ashwood P. Differential monocyte responses to TLR ligands in children with autism spectrum disorders. Brain Behav Immun 2010; 24: 64-71.

63. Shi L, Smith SE, Malkova N, Tse D, Su Y, Patterson PH. Activation of the maternal immune system alters cerebellar development in the offspring. Brain Behav Immun 2009; 23: 116-123.

64. Baksi SN, Hughes MJ. Chronic vitamin D deficiency in the weanling rat alters catecholamine metabolism in the cortex. Brain Res 1982; 242: 387-390.

65. Tenenhouse A, Warner M, Commissiong JW. Neurotransmitters in the CNS of the vitamin D deficient, hypocalcemic rat. Neurochem Int 1991; 18: 249-255.

66. Matkovits $\mathrm{T}$, Christakos S. Ligand occupancy is not required for vitamin D recepto and retinoid receptor-mediated transcriptional activation. Mol Endocrinol 1995; 9: 232-242.

67. Eyles DW, Smith S, Kinobe R, Hewison M, McGrath JJ. Distribution of the vitamin D receptor and 1 alpha-hydroxylase in human brain. J Chem Neuroanat 2005; 29: 21-30.

68. Burne TH, Becker A, Brown J, Eyles DW, Mackay-Sim A, McGrath JJ. Transient prenata Vitamin $D$ deficiency is associated with hyperlocomotion in adult rats. Behav Brain Res 2004; 154: 549-555

69. Eyles DW, Rogers F, Buller K, McGrath JJ, Ko P, French K et al. Developmental vitamin D (DVD) deficiency in the rat alters adult behaviour independently of HPA function. Psychoneuroendocrinology 2006; 31: 958-964

70. Smith MP, Fletcher-Turner A, Yurek DM, Cass WA. Calcitriol protection against dopamine loss induced by intracerebroventricular administration of 6-hydroxydopamine. Neurochem Res 2006; 31: 533-539.

71. Tekes K, Gyenge M, Folyovich A, Csaba G. Influence of neonatal vitamin A or vitamin D treatment on the concentration of biogenic amines and their metabolites in the adult rat brain. Horm Metab Res 2009; 41: 277-280.

72. Meyer U, Feldon J. Prenatal exposure to infection: a primary mechanism for abnormal dopaminergic development in schizophrenia. Psychopharmacology (Berl) 2009; 206: $587-602$.

73. Roberts RC, Roche JK, Conley RR, Lahti AC. Dopaminergic synapses in the caudate of subjects with schizophrenia: relationship to treatment response. Synapse 2009; 63: 520 530.

74. Winter C, Djodari-Irani A, Sohr R, Morgenstern R, Feldon J, Juckel G et al. Prenatal immune activation leads to multiple changes in basal neurotransmitter levels in the adult brain: implications for brain disorders of neurodevelopmental origin such as schizophrenia. Int J Neuropsychopharmacol 2009; 12: 513-524.

75. Carpenter WT, Koenig Jl. The evolution of drug development in schizophrenia: pas issues and future opportunities. Neuropsychopharmacology 2008; 33: 2061-2079. 
76. Veenstra TD, Prufer K, Koenigsberger C, Brimijoin SW, Grande JP, Kumar R. 1,25Dihydroxyvitamin D3 receptors in the central nervous system of the rat embryo. Brain Res 1998; 804: 193-205.

77. Gates MA, Torres EM, White A, Fricker-Gates RA, Dunnett SB. Re-examining the ontogeny of substantia nigra dopamine neurons. Eur J Neurosci 2006; 23: 1384-1390.

78. Smidt MP, Burbach JP. How to make a mesodiencephalic dopaminergic neuron. Nat Rev Neurosci 2007; 8: 21-32.

79. Cui X, Pelekanos M, Burne TH, McGrath JJ, Eyles DW. Maternal vitamin D deficiency alters the expression of genes involved in dopamine specification in the developing rat mesencephalon. Neurosci Lett 2010; 486: 220-223.

80. Kesby JP, Cui X, Ko P, McGrath JJ, Burne TH, Eyles DW. Developmental vitamin D deficiency alters dopamine turnover in neonatal rat forebrain. Neurosci Lett 2009; 461 : $155-158$.

81. Bayer SA, Wills KV, Triarhou LC, Ghetti B. Time of neuron origin and gradients of neurogenesis in midbrain dopaminergic neurons in the mouse. Exp Brain Res 1995; 105: 191-199.

82. Prakash N, Wurst W. Development of dopaminergic neurons in the mammalian brain. Cell Mol Life Sci 2006; 63: 187-206.

83. Meyer U, Engler A, Weber L, Schedlowski M, Feldon J. Preliminary evidence for a modulation of fetal dopaminergic development by maternal immune activation during pregnancy. Neuroscience 2008; 154: 701-709.

84. Puchacz E, Stumpf WE, Stachowiak EK, Stachowiak MK. Vitamin D increases expression of the tyrosine hydroxylase gene in adrenal medullary cells. Brain Res Mol Brain Res 1996; 36: 193-196.

85. Sanchez B, Relova JL, Gallego R, Ben-Batalla I, Perez-Fernandez R. 1,25Dihydroxyvitamin D3 administration to 6-hydroxydopamine-lesioned rats increases glial cell line-derived neurotrophic factor and partially restores tyrosine hydroxylase expression in substantia nigra and striatum. J Neurosci Res 2009; 87: 723-732.

86. Wang JY, Wu JN, Cherng TL, Hoffer BJ, Chen HH, Borlongan CV et al. Vitamin D-3 attenuates 6-hydroxydopamine-induced neurotoxicity in rats. Brain Res 2001; 904: 67-75.

87. Chen KB, Lin AM, Chiu TH. Systemic vitamin D3 attenuated oxidative injuries in the locus coeruleus of rat brain. Ann NY Acad Sci 2003; 993: 313-324, discussion 345-319.

88. Naveilhan P, Neveu I, Wion D, Brachet P. 1,25-Dihydroxyvitamin D3, an inducer of glial cell line-derived neurotrophic factor. Neuroreport 1996; 7: 2171-2175.

89. Salvatore MF, Zhang JL, Large DM, Wilson PE, Gash CR, Thomas TC et al. Striatal GDNF administration increases tyrosine hydroxylase phosphorylation in the rat striatum and substantia nigra. J Neurochem 2004; 90: 245-254.

90. Meyer U, Nyffeler M, Engler A, Urwyler A, Schedlowski M, Knuesel I et al. The time of prenatal immune challenge determines the specificity of inflammation-mediated brain and behavioral pathology. J Neurosci 2006; 26: 4752-4762.

91. Ling ZD, Potter ED, Lipton JW, Carvey PM. Differentiation of mesencephalic progenitor cells into dopaminergic neurons by cytokines. Exp Neurol 1998; 149: 411-423.

92. Potter ED, Ling ZD, Carvey PM. Cytokine-induced conversion of mesencephalic-derived progenitor cells into dopamine neurons. Cell Tissue Res 1999; 296: 235-246.

93. Carvey PM, Ling ZD, Sortwell CE, Pitzer MR, McGuire SO, Storch A et al. A clonal line of mesencephalic progenitor cells converted to dopamine neurons by hematopoietic cytokines: a source of cells for transplantation in Parkinson's disease. Exp Neurol 2001; 171: $98-108$

94. Akaneya $\mathrm{Y}$, Takahashi M, Hatanaka $\mathrm{H}$. Interleukin-1 beta enhances survival and interleukin-6 protects against MPP+ neurotoxicity in cultures of fetal rat dopaminergic neurons. Exp Neurol 1995; 136: 44-52.

95. Zhu G, Mehler MF, Mabie PC, Kessler JA. Developmental changes in progenitor cell responsiveness to cytokines. J Neurosci Res 1999; 56: 131-145.

96. Doherty GH. Developmental switch in the effects of TNFalpha on ventral midbrain dopaminergic neurons. Neurosci Res 2007; 57: 296-305.

97. Jarskog LF, Xiao H, Wilkie MB, Lauder JM, Gilmore JH. Cytokine regulation of embryonic rat dopamine and serotonin neuronal survival in vitro. Int J Dev Neurosci 1997; 15: 711-716.

98. Mehta RG, Mehta RR. Vitamin D and cancer. J Nutr Biochem 2002; 13: 252-264.

99. Meyer U, Yee BK, Feldon J. The neurodevelopmental impact of prenatal infections at different times of pregnancy: the earlier the worse? Neuroscientist 2007; 13: 241-256.

100. Ko P, Burkert R, McGrath J, Eyles D. Maternal vitamin D3 deprivation and the regulation of apoptosis and cell cycle during rat brain development. Brain Res Dev Brain Res 2004; 153: 61-68.

101. Cui X, McGrath JJ, Burne TH, Mackay-Sim A, Eyles DW. Maternal vitamin D depletion alters neurogenesis in the developing rat brain. Int J Dev Neurosci 2007; 25: 227-232.

102. Brown J, Bianco JI, McGrath JJ, Eyles DW. 1,25-Dihydroxyvitamin D3 induces nerve growth factor, promotes neurite outgrowth and inhibits mitosis in embryonic rat hippocampal neurons. Neurosci Lett 2003; 343: 139-143.

103. Joseph B, Wallen-Mackenzie A, Benoit G, Murata T, Joodmardi E, Okret S et al. p57(Kip2) cooperates with Nurr1 in developing dopamine cells. Proc Natl Acad Sci USA 2003; 100: 15619-15624

104. Wallen A, Zetterstrom RH, Solomin L, Arvidsson M, Olson L, Perlmann T. Fate of mesencephalic AHD2-expressing dopamine progenitor cells in NURR1 mutant mice. Exp Cell Res 1999; 253: 737-746.

105. Kawata H, Kamiakito T, Takayashiki N, Tanaka A. Vitamin D3 suppresses the androgenstimulated growth of mouse mammary carcinoma SC-3 cells by transcriptional repression of fibroblast growth factor 8. J Cell Physiol 2006; 207: 793-799.
106. Nyffeler M, Meyer U, Yee BK, Feldon J, Knuesel I. Maternal immune activation during pregnancy increases limbic GABAA receptor immunoreactivity in the adult offspring implications for schizophrenia. Neuroscience 2006; 143: 51-62.

107. Klafke R, Wurst W, Prakash N. Genetic control of rodent midbrain dopaminergic neuron development in the light of human disease. Pharmacopsychiatry 2008; 41(Suppl 1): S44-S50.

108. Wallen AA, Castro DS, Zetterstrom RH, Karlen M, Olson L, Ericson J et al Orphan nuclear receptor Nurr1 is essential for Ret expression in midbrain dopamine neurons and in the brain stem. Mol Cell Neurosci 2001; 18: 649-663.

109. Zetterstrom RH, Solomin L, Jansson L, Hoffer BJ, Olson L, Perlmann T. Dopamine neuron agenesis in Nurr1-deficient mice. Science 1997; 276: 248-250.

110. Kadkhodaei B, Ito T, Joodmardi E, Mattsson B, Rouillard C, Carta M et al. Nurr1 is required for maintenance of maturing and adult midbrain dopamine neurons. $J$ Neurosci 2009; 29: 15923-15932

111. Jiang C, Wan X, He Y, Pan T, Jankovic J, Le W. Age-dependent dopaminergic dysfunction in Nurr1 knockout mice. Exp Neurol 2005; 191: 154-162.

112. Solberg $Y$, Silverman WF, Pollack $Y$. Prenatal ontogeny of tyrosine hydroxylase gene expression in the rat ventral mesencephalon. Brain Res Dev Brain Res 1993; 73: 91-97.

113. Smidt MP, van Schaick HS, Lanctot C, Tremblay JJ, Cox JJ, van der Kleij AA et al. A homeodomain gene Ptx3 has highly restricted brain expression in mesencephalic dopaminergic neurons. Proc Natl Acad Sci USA 1997; 94: 13305-13310.

114. Martinat C, Bacci JJ, Leete T, Kim J, Vanti WB, Newman AH et al. Cooperative transcription activation by Nurr1 and Pitx3 induces embryonic stem cell maturation to the midbrain dopamine neuron phenotype. Proc Natl Acad Sci USA 2006; 103: 2874-2879.

115. Backman C, You ZB, Perlmann T, Hoffer BJ. Elevated locomotor activity without altered striatal dopamine contents in Nurr1 heterozygous mice after acute exposure to methamphetamine. Behav Brain Res 2003; 143: 95-100.

116. Eells JB, Lipska BK, Yeung SK, Misler JA, Nikodem VM. Nurr1-null heterozygous mice have reduced mesolimbic and mesocortical dopamine levels and increased stressinduced locomotor activity. Behav Brain Res 2002; 136: 267-275

117. Rojas $P$, Joodmardi E, Hong Y, Perlmann T, Ogren SO. Adult mice with reduced Nurr1 expression: an animal model for schizophrenia. Mol Psychiatry 2007; 12: 756-766.

118. Xing G, Zhang L, Russell S, Post R. Reduction of dopamine-related transcription factors Nurr1 and NGFI-B in the prefrontal cortex in schizophrenia and bipolar disorders. Schizophr Res 2006; 84: 36-56.

119. Bergman O, Westberg L, Nilsson LG, Adolfsson R, Eriksson E. Preliminary evidence that polymorphisms in dopamine-related transcription factors LMX1A, LMX1B and PITX3 are associated with schizophrenia. Prog Neuropsychopharmacol Biol Psychiatry 2010; 34 1094-1097.

120. Ardayfio PA, Leung A, Park J, Hwang DY, Moran-Gates T, Choi YK et al. Pitx3-deficient aphakia mice display unique behavioral responses to psychostimulant and antipsychotic drugs. Neuroscience 2010; 166: 391-396.

121. Pletnikov MV. Inducible and conditional transgenic mouse models of schizophrenia. Prog Brain Res 2009; 179: 35-47.

122. Kubo K, Tomita K, Uto A, Kuroda K, Seshadri S, Cohen J et al. Migration defects by DISC1 knockdown in C57BL/6, 129X1/SvJ, and ICR strains via in utero gene transfer and virus-mediated RNAi. Biochem Biophys Res Commun 2010; 400: 631-637.

123. Burnet PW, Anderson PN, Chen L, Nikiforova N, Harrison PJ, Wood MJ. D-amino acid oxidase knockdown in the mouse cerebellum reduces NR2A mRNA. Mol Cell Neurosci 2011; 46: 167-175

124. Blin M, Norton W, Bally-Cuif L, Vernier P. NR4A2 controls the differentiation of selective dopaminergic nuclei in the zebrafish brain. Mol Cell Neurosci 2008; 39: 592-604.

125. Furukubo-Tokunaga K. Modeling schizophrenia in flies. Prog Brain Res 2009; 179: 107-115

126. Burne T, Scott E, van Swinderen B, Hilliard M, Reinhard J, Claudianos $C$ et al. Big ideas for small brains: what can psychiatry learn from worms, flies, bees and fish? $\mathrm{Mol}$ Psychiatry 2011; 16: 7-16.

127. White $\mathrm{JH}$. Vitamin D signaling, infectious diseases, and regulation of innate immunity. Infect Immun 2008; 76: 3837-3843.

128. Cantorna MT. Vitamin $D$ and its role in immunology: multiple sclerosis, and inflammatory bowel disease. Prog Biophys Mol Biol 2006; 92: 60-64.

129. Murillo G, Nagpal V, Tiwari N, Benya RV, Mehta RG. Actions of vitamin D are mediated by the TLR4 pathway in inflammation-induced colon cancer. J Steroid Biochem Mol Biol 2010; 121: 403-407.

130. Garcion E, Sindji L, Montero-Menei C, Andre C, Brachet P, Darcy F. Expression of inducible nitric oxide synthase during rat brain inflammation: regulation by 1,25 dihydroxyvitamin D3. Glia 1998; 22: 282-294.

131. Pantelis C, Velakoulis D, McGorry PD, Wood SJ, Suckling J, Phillips LJ et al Neuroanatomical abnormalities before and after onset of psychosis: a cross-sectional and longitudinal MRI comparison. Lancet 2003; 361: 281-288.

132. Konig J. Animal models of schizophrenia. Available from: http://www.schizophreniaforum.org/ res/models/default.asp, 2009 Accessed Date 2009.

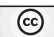

Translational Psychiatry is an open-access journal published by Nature Publishing Group. This work is licensed under the Creative Commons Attribution-Noncommercial-No Derivative Works 3.0 Unported License. To view a copy of this license, visit http://creativecommons.org/licenses/by-nc-nd/3.0/ 